Original Research

\title{
Investigations of Heavy Metals Concentrations in Leaves of Telfairia occidentalis Hook. F. (Fluted Pumpkin) in Nigeria
}

\author{
Chukwuemeka A. Nwadinigwe ${ }^{1}$, Godwin J. Udo ${ }^{1 *}$, Alfreda O. Nwadinigwe ${ }^{2}$ \\ 'Department of Pure and Industrial Chemistry, \\ ${ }^{2}$ Department of Plant Science and Biotechnology, \\ University of Nigeria, Nsukka, Nigeria \\ Received: 31 July 2014 \\ Accepted: 23 September 2014
}

\begin{abstract}
Investigations of heavy metals concentrations in leaves of Telfairia occidentalis Hook. F. (fluted pumpkin) within farmlands in Ibeno Coastal Area, Niger Delta, Nigeria was conducted using atomic absorption with a Unicam 939 spectrophotometer. The mean concentrations in $\mathrm{mg} \cdot \mathrm{kg}^{-1}$ of the heavy metals in leaves of Telfairia occidentalis were in descending order of: dry season $\mathrm{Mn}(7.73 \pm 3.06)>\mathrm{Fe}(5.93 \pm 1.28)>\mathrm{V}$ $(0.16 \pm 0.26)>\mathrm{Co}(0.10 \pm 0.01)>\mathrm{Cd}(0.21 \pm 0.16)>\mathrm{Zn}(0.03 \pm 0.02)>\mathrm{Ni}(0.02 \pm 0.01)>\mathrm{Pb}(0.02 \pm 0.01)$, and wet season $\mathrm{Mn}(7.75 \pm 3.76)>\mathrm{Fe}(5.96 \pm 4.07)>\mathrm{V}(0.21 \pm 0.09)>\mathrm{Cd}(0.19 \pm 0.06)>\mathrm{Zn}(0.11 \pm 0.07)>\mathrm{Pb}(0.09 \pm 0.15)$ $>\mathrm{Co}(0.05 \pm 0.03)>\mathrm{Ni}(0.03 \pm 0.06)$. The concentrations of the heavy metals were higher in the wet season compared to the dry season. The mean concentrations of $\mathrm{Cd}(0.205$ and 0.1699$) \mathrm{mg} \cdot \mathrm{kg}^{-1}, \mathrm{Mn}(7.729$ and 7.7531$)$ $\mathrm{mg} \cdot \mathrm{kg}^{-1}$, and $\mathrm{Fe}\left(5.93\right.$ and 5.955) $\mathrm{mg} \cdot \mathrm{kg}^{-1}$ in dry and wet seasons, respectively, exceeded WHO maximum limits of $\left(0.1 \mathrm{mg} \cdot \mathrm{kg}^{-1}\right),\left(6.0 \mathrm{mg} \cdot \mathrm{kg}^{-1}\right)$, and $\left(4.8 \mathrm{mg} \cdot \mathrm{kg}^{-1}\right)$. Heavy metal contamination in agricultural soils may lead to the disorder of soil functionality and retardation of plant growth, and influence human health through a contaminated food chain. The elevated metals levels indicated the consequences of oil-related activities as well as other sources of anthropogenic contaminants in the study area. The pollution implications of these heavy metals together with their associated health hazards on humans, farm animals, and economic crops have been discussed based on the results, international standards, controls, and available related literatures.
\end{abstract}

Keywords: heavy metals, concentration, bioaccumulation, contaminants, environment

\section{Introduction}

Environmental pollution has become an extensive and dangerous problem as a consequence of industrial and human activities. Widespread low to high pollution of large areas of agricultural land is a particular problem from indiscriminate sludge discharge from industries, crude oil pollution, and wastes [1]. Human activities have dramatically changed the balance of biochemical and geological

*e-mail: acmejames@yahoo.com cycles of many heavy metals. An assessment of environmental risk caused by soil contamination is especially important for agricultural as well as non-cultivated areas due to the fact that metals potentially harmful to human health persist in soils for a relatively long time and may transfer into the food chain in considerable amounts $[2,3]$. The persistent nature of heavy metals in soil due to their non-biodegradability raises environmental concerns because of the interdependence between plants and animals, including humans in the ecosystem [4]. Leafy vegetables tend to accumulate higher concentrations of metals 
in edible tissue compared to fruit. Vegetables can become contaminated with heavy metals if they are grown on soils contaminated by vehicular exhaust, industrial activities, and other agricultural activities, and their risk to people are of great public concern [5]. Consumption of foods contaminated with heavy metals is a major cause of health problems [6]. The levels of heavy metals (lead, cadmium, and copper) were determined in cassava samples from different locations of oil-producing areas of Delta State, Nigeria. The results indicate that the different heavy metals had higher values compared with WHO standard [7]. It has been reported that the leaves of the fluted pumpkin plants planted in farmlands far from major roads accumulate significant amounts of such heavy metals as $\mathrm{Pb}, \mathrm{Ni}, \mathrm{Cd}, \mathrm{Zn}$, $\mathrm{Mn}$, and $\mathrm{Fe}[8]$.

Telfairia occidentalis (Fluted pumpkin) is a species of cucurbitaceace family in the tropics largely consumed in Nigeria, Ghana, and Sierra Leone. The common names for fluted pumpkin include Ubong in Ibibio and Ugu in Igbo. It is a creeping vegetable that spreads low across the ground with lobed leaves and long twisting tendrils. It is a warmweather crop that grows well in low lands and tolerates elevation of some few meters above the ground. It thrives best in soils rich in organic matter [9].

Fluted pumpkin plays important role in human and livestock nutrition. It is a source of protein, oil, minerals, and vitamins. The leaves are low in crude fiber but a rich source of folic acid, calcium, zinc, potassium, cobalt, copper, iron, and vitamins $\mathrm{A}, \mathrm{C}$, and $\mathrm{K}$. It also has medicinal values as fluted pumpkin leaves and seeds could be used to increase hematological indices, improve sperm quality, and reduce blood glucose. It is rich in antioxidants, thiamin, riboflavin, and ascorbic acid. The young shoots and leaves of this vegetable are used in preparation of several delicacies in southern Nigeria, including Edikang Ikong Soup (a popular delicacy of the Efiks and Ibibios in Cross River and Akwa Ibom States, Nigeria [10]). It thrives better in the early part of the rainy season, planted between August and October, and can be grown in a garden. It can survive 3-4 years if there is moisture in the soil.

It is well known that high industrial and traffic activities contribute high levels of heavy metals to the environments. Plants grown around such areas are likely to absorb these metals either from the soil through the roots or from atmospheric contaminants through the leaves [11].

In recent times there has been an increase in industrial and domestic activities in this oil-rich region under investigation. Hence there is need for regular and systematic monitoring of the coastal area, particularly farmlands along the riverbank and some tributaries. Also, based on its persistent and cumulative nature as well as the probability of potential toxicity effects of heavy metals as a result of consumption of leafy vegetables and fruits, there is a need to test and analyze this food item to ensure that the levels of these trace elements meet the agreed international requirements. Fluted pumpkin is exceptionally important farm produce from Niger Delta where limited or no data on heavy metal contents of such highly consumed agricultural produce is unavailable even with an upsurge of industrial and domes- tic activities in the area. It is on this basis that this study was designed to determined dry and wet season concentrations of heavy metals in Telfairia occidentalis (fluted pumpkin) leaves within Farmlands in Ibeno Coastal Area, Niger Delta, Nigeria.

\section{Experimental Procedures}

\section{Study Area}

Ibeno Local Government Area has a coastal area of more than $1,200 \mathrm{~km}^{2}$. It is situated on the eastern flanks of the Niger Delta, which in turn is part of the gulf of Guinea. It is located at the southern end of Akwa Ibom State with latitude $7^{\circ} 54^{\prime}$ and $4^{\circ} 34^{\prime}$ north and longitude $7^{\circ} 54^{\prime}$ and $8^{\circ} 02^{\prime}$ east. The communities on the west bank of the Qua Iboe River do not have access to the interland except by boat through the river and creeks. Qua Iboe River estuary, which lies within the study area coordinates, has Douglas Creek emptying into it. This creek is about $900 \mathrm{~m}$ long and $8 \mathrm{~m}$ deep. It is the point where petroleum exploration and production (E and $\mathrm{P}$ ) waste from the Exxon Mobil Qua Iboe Terminal (QIT) tank farms are transferred to the lower Qua Iboe River Estuary and adjoining creeks through two $24 \mathrm{~cm}$ diameter pipes. The Exxon Mobil oily sludge dumpsite is located adjacent to this creek and the flare stack, where gas is flared continuously. Some communities in Ibeno Local Government Area are located along the Qua Iboe River while others are located on the Atlantic Littoral. Mkpanak, Upenekang, and Iwu-achang are located on the east bank of the Qua Iboe, while Okoritip and Ikot Inwang are on the west bank. IwokpomOpolom, Itak Abasi, Akete, and Okoritak are along the Atlantic coast. The Qua Iboe River estuary is in close proximity to the Exxon Mobil oil effluent treatment and discharge plant. The wastes are discharged into the Atlantic Ocean but may recede into the estuary due to tidal motion [12].

\section{Sampling Procedure}

The study area was divided into 15 sampling locations identified as (Fig. 1): 1 (Atabrikang), 2 (Ntafre), 3 (IkotInwang), 4 (Okoritip), 5 (Ukpenekang), 6 (Okpolom), 7 (IwoOkpom), 8 (Okoritip), 9 (Afia), 10 (AdahaUsuk), 11 (InuaEyetIkot), 12 (ItakAfaha), 13 (Iwokwang), 14 (Okom Ita), and 15 (Mkpanak).

Five (5) replicate samples were collected at each location and mixed together to form a composite sample representation of that location. A total of 15 composite samples of leaves of fluted pumpkin from 15 georeferenced points in 15 communities in the study area were collected and analyzed for heavy metal concentrations. The choice of plant species was based on its general growth pattern morphology and availability at the study area. The leaves of fluted pumpkin were cut using a stainless steel knife. The sampled plant was put in a clean polyethene bag, labeled, preserved in a cooler, and transported to the laboratory for pretreat- 
ment and analysis. The plant sample was identified by a taxonomist, Dr. A. U. Eshiet of the Department of Botany and Ecological Studies, University of Uyo. The voucher specimens were deposited at the herbarium of the Department of Plant Science and Biotechnology, University of Nigeria, Nsukka, Nigeria.

\section{Analytical Procedure}

Leaves of the Telfairia occendentalis samples were washed under running water to remove adhered soil particles, rinsed with deionized water to wash off dirt, and sundried to remove moisture. It was then dried in an oven at $105^{\circ} \mathrm{C}$ to constant weight, and pulverized to fine powder using a laboratory grinder and $2 \mathrm{~mm}$ mesh obtained for further analysis. The ground leaf samples were digested with $1.0 \mathrm{~cm}^{3}$ concentrated $\mathrm{HClO}_{4}, 5 \mathrm{~cm}^{3}$ concentrated $\mathrm{HNO}_{3}$, and $0.5 \mathrm{~cm}^{3}$ concentrated $\mathrm{H}_{2} \mathrm{SO}_{4}$ in a $50 \mathrm{~cm}^{3}$ Kjeldahl flask. The blank sample was prepared by repeating the same procedure but omitting the plant sample. The concentrations of $\mathrm{Ni}, \mathrm{V}, \mathrm{Cd}, \mathrm{Pb}, \mathrm{Co}, \mathrm{Fe}, \mathrm{MN}$, and $\mathrm{Zn}$ were determined using flame atomic absorption spectrophotometry (Unicam Solaar, model 969). A calibration graph was plotted for each element using measured absorbance and the corresponding concentration. The calibration curve was then used to determine the concentrations of the heavy metals [13].

\section{Results}

The results of mean concentrations in $\mathrm{mg} \cdot \mathrm{kg}^{-1}$ of the heavy metals ( $\mathrm{Ni}, \mathrm{V}, \mathrm{Cd}, \mathrm{Pb}, \mathrm{Mn}, \mathrm{Fe}, \mathrm{Co}$, and $\mathrm{Zn}$ ) in leaves of Telfairia occidentalis samples from the Ibeno Coastal Area during dry and wet seasons are presented in Tables 13 . The values are given as mean \pm standard deviation.

\section{Discussion of Results}

Trends in the distribution of the mean concentrations in $\mathrm{mg} \cdot \mathrm{kg}^{-1}$ of the heavy metals in leaves of Telfairia occidentalis samples for dry and wet seasons were: dry season $\mathrm{Mn}$ $(7.73 \pm 3.06)>\mathrm{Fe}(5.93 \pm 1.28)>\mathrm{V}(0.16 \pm 0.26)>\mathrm{Co}$ $(0.10 \pm 0.01)>\mathrm{Cd}(0.21 \pm 0.16)>\mathrm{Zn}(0.03 \pm 0.02)>\mathrm{Ni}$ $(0.02 \pm 0.01)>\mathrm{Pb}(0.02 \pm 0.01)$, and wet season $\mathrm{Mn}$ $(7.75 \pm 3.76)>\mathrm{Fe}(5.96 \pm 4.07)>\mathrm{V}(0.21 \pm 0.09)>\mathrm{Cd}$ $(0.19 \pm 0.06)>\mathrm{Zn}(0.11 \pm 0.07)>\mathrm{Pb}(0.09 \pm 0.15)>\mathrm{Co}$ $(0.05 \pm 0.03)>\mathrm{Ni}(0.03 \pm 0.06)$ (Tables 1 and 2$)$. The investigation showed that the mean concentrations of some of the heavy metals analyzed were within the normal concentration range of heavy metals in vegetables as recorded by Alloway [16], WHO/FAO, Federal Ministry of Environment, and Nigeria and German Federal Health Agency guidelines for heavy metals in vegetables and foodstuffs (Table 3).

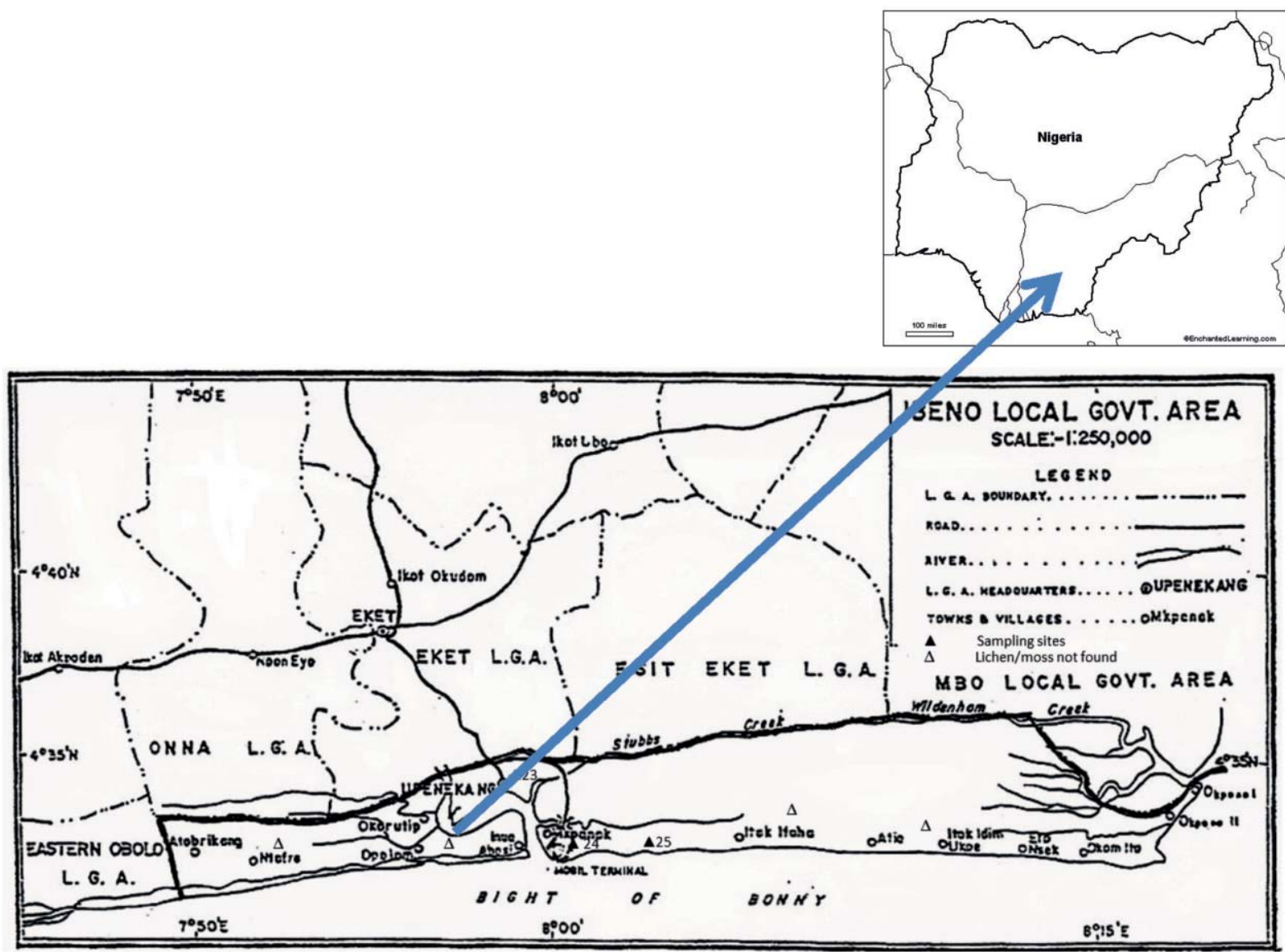

Fig. 1. Map of the study area showing sampling locations.

Source: Survey and Geo-informatics Department Ministry of Lands and Housing, Akwa Ibom State. 


\begin{tabular}{|c|c|c|c|c|c|c|c|c|}
\hline 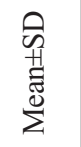 & 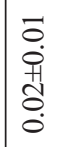 & 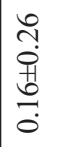 & 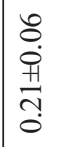 & 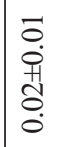 & 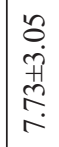 & 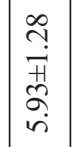 & $\begin{array}{l}0 \\
0 \\
0 \\
0 \\
0 \\
0 \\
0\end{array}$ & 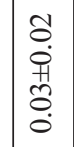 \\
\hline 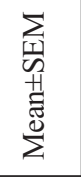 & 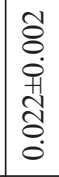 & $\begin{array}{l}\vec{J} \\
0 \\
0 \\
+1 \\
\text { d } \\
0 \\
0\end{array}$ & 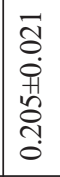 & 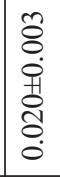 & 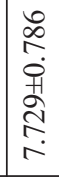 & 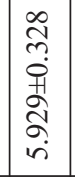 & $\begin{array}{l}\text { fo } \\
\text { fo } \\
0 \\
0 \\
0 \\
0 \\
0\end{array}$ & 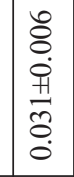 \\
\hline $\overrightarrow{\partial^{\circ}}$ & $\begin{array}{l}\text { aे. } \\
\text { aे }\end{array}$ & \begin{tabular}{l}
$\infty$ \\
$\infty$ \\
\hdashline \\
2
\end{tabular} & 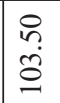 & ঙ̊. & 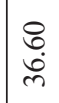 & $\begin{array}{l}\stackrel{B}{\vec{\lambda}} \\
\vec{\sim}\end{array}$ & $\begin{array}{l}\stackrel{R}{i} \\
\text { d. }\end{array}$ & $\begin{array}{l}8 \\
8 \\
\infty\end{array}$ \\
\hline 己 & ஓ্ণ & $\stackrel{\infty}{\circ}$ & 華 & 尺ै & ڤ̊ & $\begin{array}{l}0 \\
\\
0\end{array}$ & $\underset{\widehat{\sigma}}{\widehat{\sigma}}$ & $\begin{array}{l}0 \\
0 \\
0 \\
0 \\
0\end{array}$ \\
\hline คิ & $\overrightarrow{0}$ & 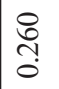 & $\begin{array}{l}\mathscr{0} \\
\ddot{0} \\
\stackrel{0}{0}\end{array}$ & $\stackrel{\Delta}{0}$ & $\begin{array}{l}\tilde{n} \\
\text { co } \\
\dot{m}\end{array}$ & 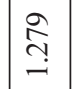 & 声 & बे. \\
\hline$\stackrel{\text { ळี }}{\Sigma}$ & तิ & $\frac{6}{0}$ & 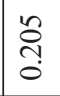 & 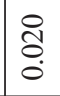 & 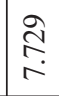 & $\begin{array}{l}\text { 亏े } \\
\text { iे }\end{array}$ & 흥 & $\vec{\delta}$ \\
\hline$\hat{\Xi}$ & 官 & ठै. & 吾 & $\stackrel{m}{a}$ & $\begin{array}{l}\infty \\
\infty \\
n \\
n \\
n\end{array}$ & $\begin{array}{l}\vec{n} \\
0 \\
0\end{array}$ & $\overrightarrow{0}$ & $\ddot{\circ}$ \\
\hline$a^{\frac{2}{3}}$ & ڤิ & $\stackrel{\infty}{\stackrel{\infty}{0}}$ & 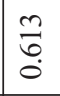 & $\stackrel{\Delta}{\stackrel{0}{0}}$ & $\stackrel{\infty}{\stackrel{\infty}{=}}$ & $\begin{array}{l}\text { ठे } \\
\stackrel{+}{+}\end{array}$ & $\overrightarrow{\bar{\sigma}}$ & : \\
\hline$\stackrel{ \pm}{ \pm}^{ \pm}$ & $\begin{array}{l}\widehat{\widehat{\sigma}} \\
\stackrel{0}{0}\end{array}$ & $\stackrel{m}{=}$ & $\stackrel{\infty}{\circ}$ & 产 & $\begin{array}{l}\hat{\alpha} \\
\hat{\sigma} \\
\end{array}$ & $\mid \begin{array}{c}\tilde{a} \\
\text { ô } \\
\dot{+}\end{array}$ & $\frac{n}{0}$ & $\overrightarrow{5}$ \\
\hline$\stackrel{\varrho}{9}$ & ڤి & $\stackrel{n}{\circ}$ & $\stackrel{n}{0}$ & $\stackrel{\sigma}{\circ}$ & $\begin{array}{l}\stackrel{\Omega}{\infty} \\
\stackrel{\infty}{=} \\
=\end{array}$ & $\begin{array}{l}\vec{b} \\
\stackrel{+}{+}\end{array}$ & $\vec{\infty}$ & ठ̊. \\
\hline$\stackrel{\beth}{9}$ & 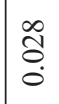 & $\stackrel{\infty}{\stackrel{0}{0}}$ & $\frac{2}{n}$ & 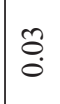 & $\begin{array}{l}\hat{\alpha} \\
\hat{\sigma}\end{array}$ & 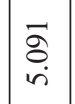 & 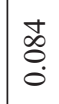 & 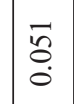 \\
\hline 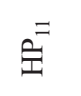 & ¿̂े & $\hat{o}$ & 合 & Oి & $\begin{array}{l}\hat{\infty} \\
\stackrel{\Xi}{\Xi}\end{array}$ & 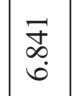 & $\stackrel{\infty}{0}$ & $\stackrel{0}{0}$ \\
\hline$\stackrel{\varrho}{9}$ & ֻั: & 容 & 方 & 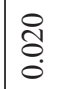 & $\stackrel{n}{\stackrel{n}{n}}$ & 官 & \begin{tabular}{l}
0 \\
\multirow{0}{0}{} \\
0
\end{tabular} & के. \\
\hline$\hat{\Xi}$ & స్ర & 悹 & 至 & ठे. & 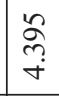 & $\begin{array}{l}\overrightarrow{\vec{D}} \\
\dot{b} \\
\end{array}$ & $\overrightarrow{5}$ & 号 \\
\hline$\sharp^{\infty}$ & ్ֶరి & $\overline{8}$ & 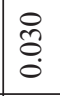 & 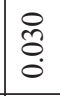 & $\begin{array}{l}\vec{\partial} \\
\dot{\sigma} \\
\dot{i}\end{array}$ & $\frac{\hat{n}}{6}$ & $\begin{array}{l}8 \\
0 \\
0 \\
0\end{array}$ & 咅 \\
\hline$\hat{\Xi}$ & $\vec{\circ}$ & $\overrightarrow{\widetilde{\delta}}$ & 尽 & $\vec{G}$ & 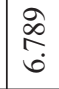 & $\begin{array}{l}\infty \\
\hat{\sigma} \\
\text { in }\end{array}$ & $\overleftarrow{0}$ & \\
\hline झే & ठิ & $\stackrel{0}{0}$ & 宍 & ठ̊. & $\begin{array}{l}\hat{a} \\
\hat{\tilde{n}} \\
\hat{n}\end{array}$ & 官 & $\hat{\delta}$ & ¿ \\
\hline$\nexists^{n}$ & ठे. & 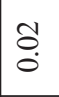 & 㠻 & 宊 & 字 & $\begin{array}{l}\vec{\sigma} \\
\dot{\sigma} \\
\dot{0}\end{array}$ & $\stackrel{0}{0}$ & $\hat{z}$ \\
\hline$\xi^{+}$ & तิ & ô. & 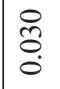 & 宊 & $\begin{array}{l}n \\
n \\
n \\
n\end{array}$ & $\begin{array}{l}\infty \\
\infty \\
i \\
i \\
\text { in }\end{array}$ & $\begin{array}{l}\infty \\
0 \\
0 \\
0\end{array}$ & $\overrightarrow{\tilde{\sigma}}$ \\
\hline$\hat{\nexists}$ & ڤ్రి & $\stackrel{n}{\tilde{o}}$ & $\stackrel{m}{0}$ & 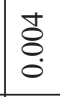 & 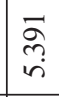 & $\begin{array}{l}\infty \\
\hat{\sigma} \\
\text { in }\end{array}$ & है. & 范 \\
\hline$\xi^{N}$ & $\hat{\check{o}}$ & $\stackrel{1}{\stackrel{0}{0}}$ & $\frac{8}{0}$ & 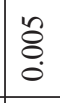 & 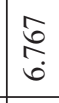 & $\begin{array}{l}n \\
\hat{o} \\
\infty\end{array}$ & $\vec{r}$ & ठे \\
\hline$\hat{\Xi}$ & ôे. & $\begin{array}{l}8 \\
0 \\
0\end{array}$ & 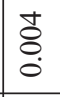 & 苛 & ڤેे & $\begin{array}{c}0 \\
\tilde{\delta} \\
\dot{+} \\
\dot{0}\end{array}$ & 守े & 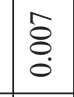 \\
\hline 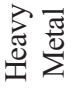 & $\bar{z}$ & $>$ & $\overrightarrow{0}$ & $\overrightarrow{2}$ & 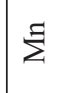 & $\begin{array}{l}0 \\
0\end{array}$ & 8 & งี \\
\hline
\end{tabular}

\begin{tabular}{|c|c|c|c|c|c|c|c|c|}
\hline 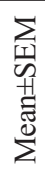 & $\begin{array}{l}\text { ڤे } \\
0 \\
+1 \\
\infty \\
0 \\
0 \\
0\end{array}$ & 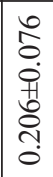 & $\mid \begin{array}{c}4 \\
0 \\
0 \\
0 \\
0 \\
+1 \\
0 \\
0 \\
0\end{array}$ & $\begin{array}{l}\text { na } \\
0 \\
0 \\
0 \\
0 \\
0 \\
0 \\
0\end{array}$ & 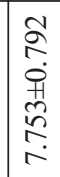 & $\begin{array}{l}\tilde{n} \\
\hat{o} \\
\dot{+1} \\
\hat{n} \\
\hat{\sigma} \\
i\end{array}$ & 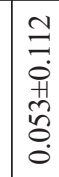 & $\begin{array}{c}n \\
n \\
0 \\
0 \\
1 \\
0 \\
0 \\
0\end{array}$ \\
\hline 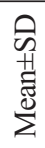 & $\begin{array}{l}\text { ñ. } \\
\text { in } \\
\text { o. } \\
\stackrel{0}{0}\end{array}$ & 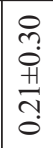 & $\begin{array}{l}\overrightarrow{\vec{v}} \\
\hat{0} \\
\stackrel{+}{1} \\
\dot{0}\end{array}$ & $\begin{array}{l}\infty \\
0 \\
0 \\
0 \\
0 \\
0 \\
0 \\
0\end{array}$ & $\begin{array}{l}\hat{\sigma} \\
\dot{p} \\
\stackrel{H}{2} \\
\stackrel{2}{r}\end{array}$ & 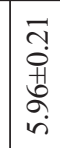 & 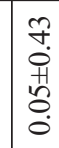 & 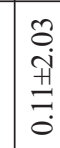 \\
\hline $\begin{array}{l}\text { 己े } \\
0^{\circ}\end{array}$ & $\frac{\text { So }}{\dot{m}}$ & 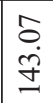 & \begin{tabular}{l}
0 \\
$n$ \\
\hdashline \\
\hdashline
\end{tabular} & $\begin{array}{l}\hat{a} \\
\infty \\
i n\end{array}$ & $\begin{array}{l}\dot{8} \\
\dot{m}\end{array}$ & $\begin{array}{l}\infty \\
\infty \\
\stackrel{i}{ }\end{array}$ & $\begin{array}{l}\text { 寸 } \\
\dot{f}\end{array}$ & 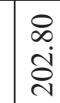 \\
\hline z & $\frac{0}{n}$ & 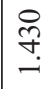 & 岇 & \begin{tabular}{|l}
$\vec{\infty}$ \\
$\hat{0}$ \\
0
\end{tabular} & $\begin{array}{l}0 \\
\text { ஸे } \\
0\end{array}$ & 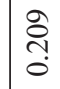 & 売 & $\begin{array}{l}\infty \\
\delta \\
\delta \\
i\end{array}$ \\
\hline की & $\stackrel{n}{\circ}$ & ๙ิ ڤે & $\stackrel{0}{\grave{1}}$ & है & $\begin{array}{l}R \\
0 \\
\dot{r}\end{array}$ & 啇 & 竎 & $\begin{array}{l}\infty \\
\text { ర్j }\end{array}$ \\
\hline
\end{tabular}

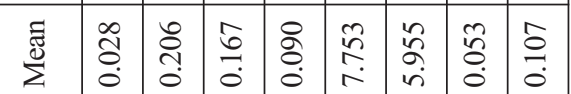

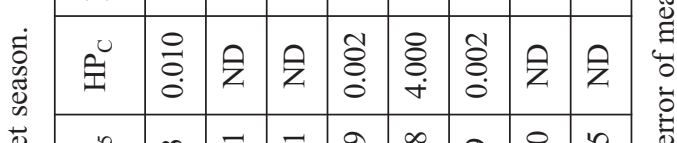

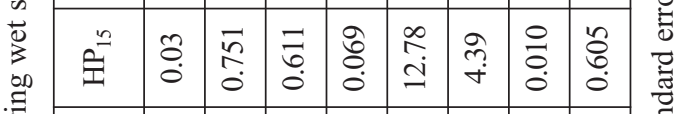

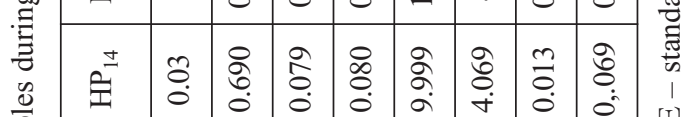
$\frac{0}{2}$ 营 $\cong$ วิ

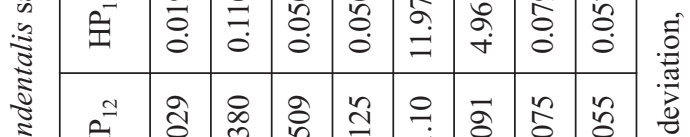

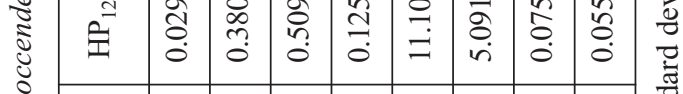

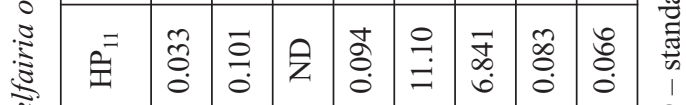

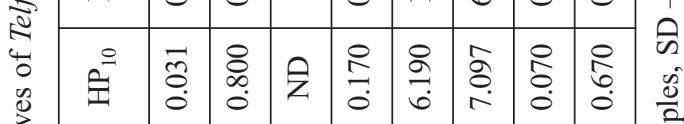

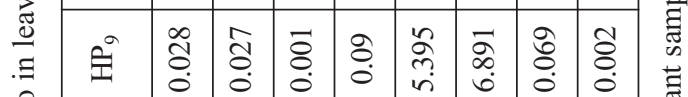

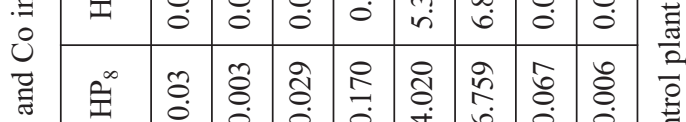
สี

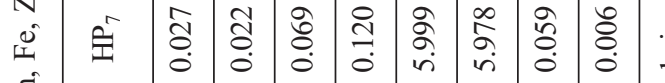
हี 0 ๑

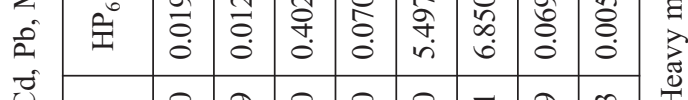
王

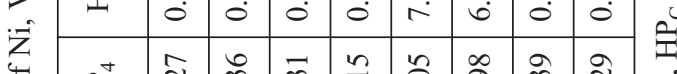

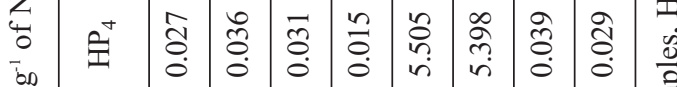
安

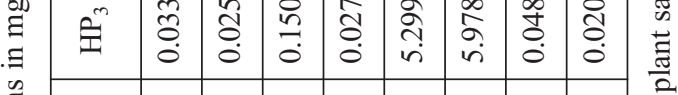

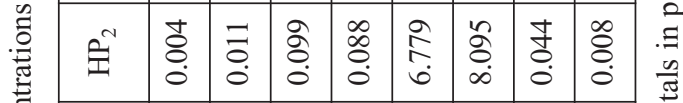
䓌

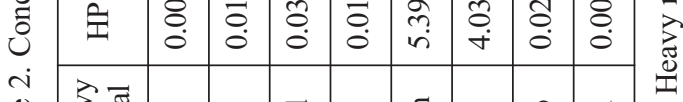

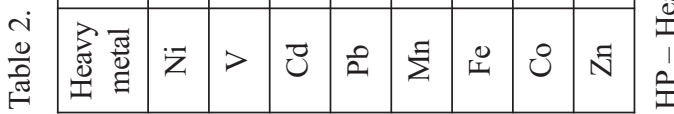


Table 3. Average concentrations in $\mathrm{mg} \cdot \mathrm{kg}^{-1}$ of heavy metals for wet and dry seasons in leaves of Telfairia occendentalis with WHO standard.

\begin{tabular}{|c|c|c|c|c|}
\hline $\begin{array}{c}\text { Heavy } \\
\text { metals }\end{array}$ & $\begin{array}{c}\text { Mean for } \\
\text { dry } \\
\text { season }\end{array}$ & $\begin{array}{c}\text { Mean for } \\
\text { wet } \\
\text { season }\end{array}$ & $\begin{array}{c}\text { Average } \\
\text { concentrations } \\
\text { for dry and wet } \\
\text { seasons }\end{array}$ & $\begin{array}{c}\text { WHO/FAO } \\
\text { standards }\end{array}$ \\
\hline $\mathrm{Ni}$ & 0.0221 & 0.028 & 0.025 & 1.0 \\
\hline $\mathrm{V}$ & 0.016 & 0.206 & 0.111 & 1.0 \\
\hline $\mathrm{Cd}$ & 0.205 & 0.199 & 0.202 & 0.1 \\
\hline $\mathrm{Pb}$ & 0.020 & 0.090 & 0.055 & 0.1 \\
\hline $\mathrm{Mn}$ & 7.729 & 7.753 & 7.741 & 6.0 \\
\hline $\mathrm{Fe}$ & 5.9305 & 5.955 & 5.943 & 4.8 \\
\hline $\mathrm{Co}$ & 0.101 & 0.053 & 0.077 & 7.0 \\
\hline $\mathrm{Zn}$ & 0.031 & 0.605 & 0.318 & 6.0 \\
\hline
\end{tabular}

FAO/WHO standards from: Cordex Alimentirus Commission (Joint FAO/WHO Food Standards Programme Codex Committee on Contaminants in Foods) $[14,15]$.

FAO - Food and Agricultural Organization of United Nations, WHO - World Health Organization

The exceptions were the mean concentrations of $\mathrm{Cd}$ (0.205 and 0.1699) $\mathrm{mg} \cdot \mathrm{kg}^{-1}, \mathrm{Mn}\left(7.729\right.$ and 7.7531) $\mathrm{mg} \cdot \mathrm{kg}^{-1}$, and $\mathrm{Fe}$ (5.93 and 5.955) $\mathrm{mg} \cdot \mathrm{kg}^{-1}$, which exceeded WHO/FAO maximum limit of $\left(0.1 \mathrm{mg} \cdot \mathrm{kg}^{-1}\right),\left(6.0 \mathrm{mg} \cdot \mathrm{kg}^{-1}\right)$, and $\left(4.8 \mathrm{mg} \cdot \mathrm{kg}^{-1}\right)$ for dry and wet seasons, respectively (Table 3). The concentrations of heavy metals in leaves of fluted pumpkin samples were higher in wet season when compared with the dry season counterpart. This could be due to increases in soil water level, which resulted in increased assimilation and translocation of nutrients by the plant. The seasonal differences in the heavy metals concentrations in fluted pumpkin might also be due to the soil compositions, $\mathrm{pH}$, and the rate of uptake of minerals by the plant. This is contrary to the report of Oladunni et al., who reported lower concentrations of heavy metals in wet season compared to dry season [3]. In a related work by Essiet et al. [17], the order of bioaccumulation of heavy metals was $\mathrm{Mn}>\mathrm{Fe}>\mathrm{Zn}>\mathrm{Li}>\mathrm{Co}>\mathrm{Pb}>\mathrm{Mn}>\mathrm{Cd}>\mathrm{V}>\mathrm{Hg}>$ $\mathrm{Cu}=\mathrm{Sc}>\mathrm{Cr}$.

Also, in research by Nwajei et al. [17], select metals such as lead, copper, cadmium, nickel, zinc, chromium, manganese, arsenic, iron, selenium, and cobalt respectively were analyzed and were all detected in plant samples from the study area. In the same way, an investigation by Uboh et al. [9] showed that the levels of heavy metals $(\mathrm{Pb}, \mathrm{Ni}$, $\mathrm{Mn}, \mathrm{Cd}$, and $\mathrm{Zn}$ ) were significantly higher than the recommended values in fluted pumpkin seed harvested from the study area. Similarly, Edem et al. [18], in a related study titled "Distribution of heavy metals in leaves, stems and roots of fluted pumpkin in Calabar," elevated levels of Fe $\left(3.14 \mathrm{mg} \cdot \mathrm{kg}^{-1}\right)>\mathrm{Cu}\left(0.88 \mathrm{mg} \cdot \mathrm{kg}^{-1}\right)>\mathrm{Mn}\left(0.40 \mathrm{mg} \cdot \mathrm{kg}^{-1}\right)>$ $\mathrm{Pb}\left(0.13 \mathrm{mg} \cdot \mathrm{kg}^{-1}\right)>\mathrm{Cr}\left(0.08 \mathrm{mg} \cdot \mathrm{kg}^{-1}\right)>\mathrm{Zn}\left(0.04 \mathrm{mg} \cdot \mathrm{kg}^{-1}\right)$ were recorded.
Dietary exposure to heavy metals, namely cadmium $(\mathrm{Cd})$, lead $(\mathrm{Pb})$, zinc $(\mathrm{Zn})$, and copper $(\mathrm{Cu})$, has been identified as a risk to human health through the consumption of vegetable crops. Heavy metals affect the nutritive values of agricultural materials and have deleterious effects on human beings. National and international regulations on food quality set the maximum permissible levels of toxic metals in human food; hence the important aspect of food quality should be to control the concentrations of these heavy metals in food. Osu and Ogoko [6] stated that Verninia Amydalin, Telfairia occidentalis, and Amarathus are good accumulators of heavy metals. Toxic metals may be absorbed by vegetables through several processes and finally enter the food chain at high concentrations capable of causing serious health risks to consumers. Their toxicity can damage or reduce mental and central nervous function, lower energy levels, and damage blood composition, lungs, kidneys, liver, and other vital organs.

\section{Seasonal Dynamics of Individual Elements}

$$
\text { Nickel (Ni) }
$$

The mean \pm SD $\left(0.0221 \pm 0.0108 \mathrm{mg} \cdot \mathrm{kg}^{-1}\right)$ and $\% \mathrm{CV}$ (49.015\%) of $\mathrm{Ni}$ were recorded in Telfairia occidentalis leaves in the dry season. In the wet season mean \pm SD $\left(0.0283 \pm 0.0146 \mathrm{mg} \cdot \mathrm{kg}^{-1}\right)$ and \% CV (51.625\%) were also documented (Tables 1 and 2). The total average concentration of Ni in leaves of Telfairia occidentalis of the study area was $0.03 \mathrm{mg} \cdot \mathrm{kg}^{-1}$ (Table 3 and Fig. 2) greater than the control sample, but within the normal range of WHO standard for heavy metals in vegetables. The highest concentration of $0.037 \mathrm{mg} \cdot \mathrm{kg}^{-1}$ and lowest $0.001 \mathrm{mg} \cdot \mathrm{kg}^{-1}$ was recorded in locations 1 (Atabrikang) and 11 (Inua Eyet Ikot). Gideon et al. [8] reported a mean concentration of $0.065 \mathrm{mg} \cdot \mathrm{kg}^{-1} \mathrm{Ni}$. Similarly, Nwajei et al. [19] reported $1.83 \pm 0.06 \mathrm{mg} \cdot \mathrm{kg}^{-1}$ and Sobukola et al. [20] observed $0.24 \pm 0.01 \mathrm{mg} \cdot \mathrm{kg}^{-1} \mathrm{Ni}$ in leafy vegetable. Okra absorbed more $\mathrm{Fe}, \mathrm{Ni}$, and $\mathrm{Cd}$. In research by Agbogidi [4], "Trace metal profile of some fruits in Kokori and Abraka Market, Delta State, Nigeria,' the results indicated the presence of elevated trace elements, including lead, chromium, cadmium, zinc, manganese, nickel, cobalt, and copper in the test

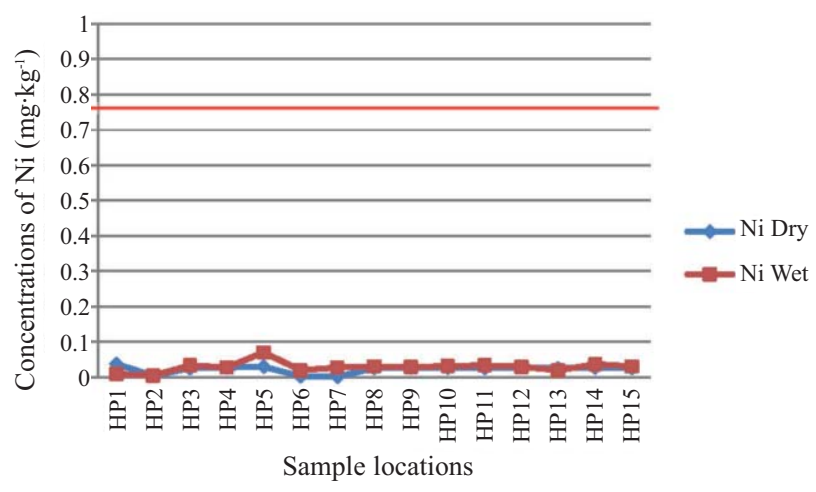

Fig. 2. Dry and wet season variations in concentrations of $\mathrm{Ni}$ in leaves of Telfairia occidentalis with WHO threshold. HP1 to HP15 - sample locations 
fruits from Kokori market when compared with values obtained from the same fruits obtained from Abraka (a nonoil producing community) in the same local government. Trace metals play an essential biological role in plant and human metabolisms; introduction of crude oil into agricultural soil adversely and severely inhibits agronomic growth and development of Abelmoschus esculentus and reduces its height and girth [21-23]. Plants experience oxidative stress upon exposure to heavy metals that leads to cellular damage [24]. Nickel toxicity in plants is characterized by chlorosis and necrosis of leaves. Also, stunting of the roots, deformation of various plant organs, and wilting are possible. Ni can cause various kinds of cancer on different organs within the bodies of animals.

\section{Vanadium $(V)$}

The mean concentration of $\mathrm{V}$ and $\% \mathrm{CV}$ of $\mathrm{V}$ in leaves of Telfairia occidentalis were $0.16 \pm 0.26 \mathrm{mg} \cdot \mathrm{kg}^{-1}$ and $159.8 \%$ in dry season (Table 1, Fig. 3). Also, mean concentration $\left(0.206 \pm 0.295 \mathrm{mg} \cdot \mathrm{kg}^{-1}\right)$ and \% CV (143.072\%) of V were obtained in leaves of Telfairia occidentalis samples in the wet season (Table 2, Fig. 2). Total average of V for dry and wet seasons was $0.111 \mathrm{mg} \cdot \mathrm{kg}^{-1}$ vanadium (Table 3 ). Mean concentration of $\mathrm{V}\left(0.111 \mathrm{mg} \cdot \mathrm{kg}^{-1}\right)$ in leaves of Telfairia occedentalis was within WHO/FAO $\left(1.0 \mathrm{mg} \cdot \mathrm{kg}^{-1}\right)$, and Federal Ministry of Environment, Nigeria maximum threshold limits. Vanadium was not detected in leaves of Telfairia occedentalis in background samples (Tables 1 and 2). The highest concentration of $0.751 \mathrm{mg} \cdot \mathrm{kg}^{-1}$ was obtained at location 15 (Mkpanak) and the lowest concentration of $0.001 \mathrm{mg} \cdot \mathrm{kg}^{-1}$ at location 8 (Okoritip). Several animal and human studies have suggested that vanadium may lower blood sugar levels and improve sensitivity to insulin in people with type 2 diabetes. In one study of people with type 2 diabetes, vanadium also lowered their total cholesterol [25]. Vanadium cannot be destroyed in the environment. It can only change its form or become attached or separated from airborne particulate, soil, particulate in water, and sediment. Damage to the lungs, throat, and nose have been observed in rats and mice exposed to vanadium pentoxide. Nausea, mild diarrhea, and stomach cramps have been reported in people. Lung cancer has been found in mice exposed to vanadium pentoxide. The International Agency for Research on Cancer (IARC) has determined that vanadium is possibly carcinogenic to humans. The acute effects of vanadium are irritation of lungs, throat, eyes, and nasal cavities (other health effects of vanadium uptake can be found in [25]).

\section{Cadmium (Cd)}

The mean concentrations of Cd in leaves Telfairia occidentalis samples were $0.205 \pm 0.21 \mathrm{mg} \cdot \mathrm{kg}^{-1}$ and $0.170 \pm 0.21$ $\mathrm{mg} \cdot \mathrm{kg}^{-1}$ for dry and wet seasons, respectively (Tables $1-2$, Fig. 4). The total average concentration of $\mathrm{Cd}$ in leaves of Telfairia occidentalis planted in the study area was 0.202 $\mathrm{mg} \cdot \mathrm{kg}^{-1}$ (Table 3 ). The mean concentrations for dry and wet

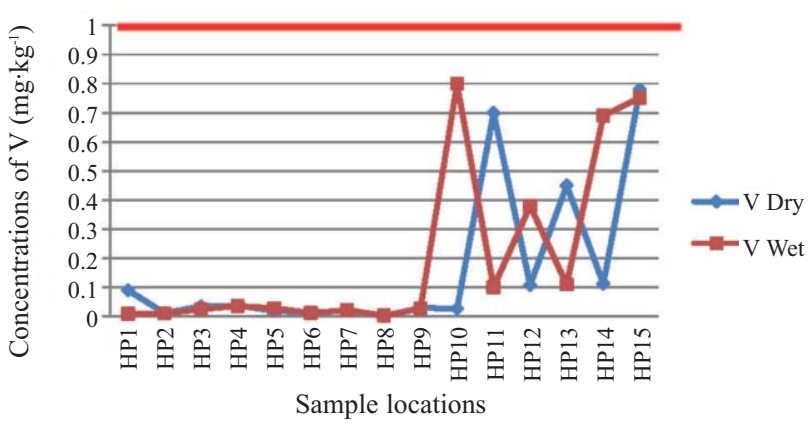

Fig. 3. Dry and wet season variations in concentrations of $\mathrm{V}$ in leaves of Telfairia occidentalis with WHO threshold.

seasons and the total average concentrations of $\mathrm{Cd}$ in leaves of Telfairia occidentalis exceeded the $0.1 \mathrm{mg} \cdot \mathrm{kg}^{-1}$ guideline value recommended by $\mathrm{WHO} / \mathrm{FAO}$ and the German Federal Agency for vegetables and foodstuffs. Cadmium was not detected in leaves of Telfairia occedentalis in background samples (Tables 1 and 2). The highest concentration of $0.613 \mathrm{mg} \cdot \mathrm{kg}^{-1}$ and the lowest concentration of 0.004 $\mathrm{mg} \cdot \mathrm{kg}^{-1}$ were recorded in plant samples from locations 15 (Mkpanak) and 1 (Atabrikang), respectively.

Cadmium was not detected in plant samples of locations 9, 10, and 11. Asualo et al. [26] recorded mean concentrations of $0.05 \mathrm{mg} \cdot \mathrm{kg}^{-1} \mathrm{Cd}$ and $\% \mathrm{CV} 27 \%$ in their research work. Also, Essiet et al. [17] obtained a mean concentration of $0.18 \mathrm{mg} \cdot \mathrm{kg}^{-1} \mathrm{Cd}$ in plants grown in oil-polluted soil. Similarly, Howard et al. [27] documented a mean concentration of $1.12 \mathrm{mg} \cdot \mathrm{kg}^{-1} \mathrm{Cd}$ in Mkpanak, Ibeno Local Government Area. A related work by Nwajei et al. [19] observed $0.01 \pm 0.00 \mathrm{mg} \cdot \mathrm{kg}^{-1} \mathrm{Cd}$ in tomato fruit harvested from the study area. Kihampa et al. [5] stated that the levels of $\mathrm{Zn}, \mathrm{Cr}, \mathrm{Pb}$, and $\mathrm{Cd}$ were above the permissible levels of heavy metals in food as outlined in WHO guidelines and the Tanzania Bureau of Standards (TBS). Also, in a related work by Adekunle et al. [28], Cd phytotoxicity was shown by growth retardation, reduction in nutrient values, and photosynthetic pigments. Ogboi [1] stated that the concentration of cadmium and lead in both the root and stem of groundnuts have exceeded permissible levels for human and animal consumption as recommended by FAO and WHO. The concentrations of heavy metals in groundnuts were in the order of $\mathrm{Cd}>\mathrm{Ni}>\mathrm{Pb}>\mathrm{Fe}>\mathrm{Zn}$ [29]. Intake of cadmium-contaminated food causes acute gastrointestinal effects, such as vomiting and diarrhea, kidney damage is the main problem for patients chronically exposed to cadmium, and maternal exposure to cadmium is associated with low birth weight and an increase in spontaneous abortions [30].

\section{Lead $(\mathrm{Pb})$}

The mean concentration and \% $\mathrm{CV}$ of $\mathrm{Pb}$ in leaves of Telfairia occidentalis samples in the soil of study area were $0.02 \pm 0.085 \mathrm{mg} \cdot \mathrm{kg}^{-1}$ and $108.546 \%$ respectively for dry season (Table 1, Fig. 5). During the wet season, mean concentration $\left(0.0902 \pm 0.0524 \mathrm{mg} \cdot \mathrm{kg}^{-1}\right)$ and \% CV $(58.066 \%)$ were recorded in leaves of Telfairia occidentalis samples in the 
soil of the study area (Table 2, Fig. 5). The total average concentration of $0.055 \mathrm{mg} \cdot \mathrm{kg}^{-1}$ was obtained in leaves of fluted pumpkin samples planted in the soil of the study area for dry and wet seasons (Table 3). The mean concentrations were slightly higher than the levels of $\mathrm{Pb}$ in the control plant sample, but within WHO standards $\left(0.1 \mathrm{mg} \cdot \mathrm{kg}^{-1}\right)$ for $\mathrm{Pb}$ in vegetables and $0.25 \mathrm{mg} \cdot \mathrm{kg}^{-1}$ guideline values in foodstuffs by Nigeria's Federal Ministry of the Environment. The concentration of $\mathrm{Pb}$ obtained in this investigation is low compared to the concentration of $4.31 \pm 0.37 \mathrm{mg} \cdot \mathrm{kg}^{-1}$ recorded by Opajobi et al. [31]. Based on the results obtained in this research, leaves of Telfairia occidentalis are not under risk of pollution by lead. Though free from lead contamination, soil analysis before planting is encouraged since $\mathrm{Pb}$ is not completely absent. In a similar investigation by Essiett et al. [17], a mean concentration of $0.36 \mathrm{mg} \cdot \mathrm{kg}^{-1}$ $\mathrm{Pb}$ was recorded higher than the $0.02 \mathrm{mg} \cdot \mathrm{kg}^{-1}$ recorded in this study. Kihampa et al. [5] reported (0.49 and 20.65) $\mathrm{mg} \cdot \mathrm{kg}^{-1}$ of lead in vegetable. Also, Nwejei et al. [19] obtained $4.01 \pm 0.05 \mathrm{mg} \cdot \mathrm{kg}^{-1}$ and $(2.96 \pm 0.25) \mathrm{mg} \cdot \mathrm{kg}^{-1}$ of $\mathrm{Pb}$ in tomato leaves and fruit samples in the study area. Asualu et al. [26] revealed mean concentrations of $0.07 \mathrm{mg} \cdot \mathrm{kg}^{-1}$, $0.06 \mathrm{mg} \cdot \mathrm{kg}^{-1}$ and $0.07 \mathrm{mg} \cdot \mathrm{kg}^{-1}$ of $\mathrm{Pb}$ in root stems and leaf samples, respectively, in leafy vegetables. Green leafy vegetables may absorb lead from soil where exposure is high [31].

Anthropogenic sources of heavy metal contaminants include burning of fossil fuel, oil spills, and the use of fertilizers that contain heavy metals. This can lead to increased

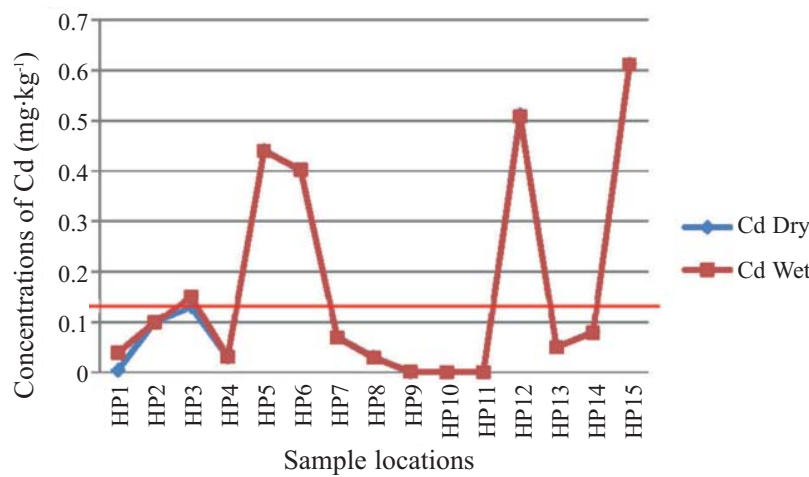

Fig. 4. Dry and wet season variations in concentrations of $\mathrm{Cd}$ in leaves of Telfairia occidentalis with WHO threshold.

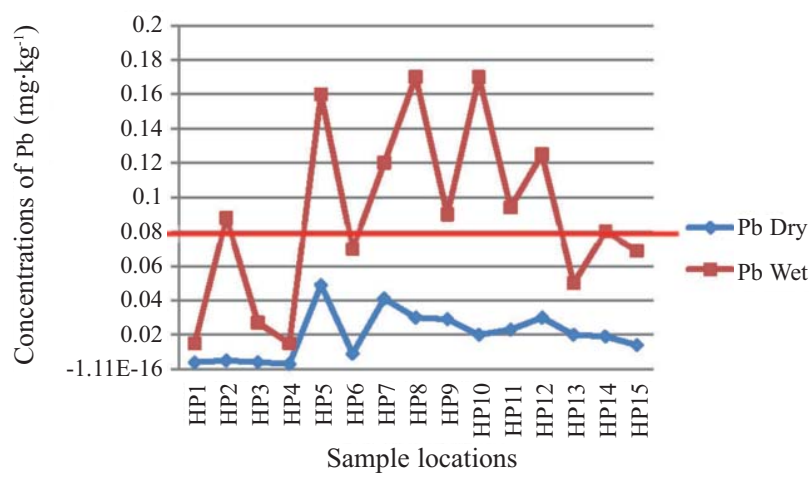

Fig. 5. Dry and wet season variations in concentrations of $\mathrm{Pb}$ in leaves of Telfairia occidentalis with WHO threshold. heavy metals load in the edible tissues of the plant and, consequently, create a pathway for heavy metal accumulation for people who consume such plants. In general, plants do not absorb or accumulate lead. However, in soils high in lead it is possible for some lead to be taken up. In children, lead is most damaging at the age of six years and younger. At low levels lead can be harmful and is associated with: learning disabilities resulting in decreased intelligence, attention deficit disorder, behavior issues, nervous system damage, speech and language impairment, decreased muscle growth, decreased bone growth, and kidney damage [30].

\section{Manganese (Mn)}

The mean concentration and \% CV of $\mathrm{Mn}$ in leaves of Telfairia occidentalis samples in the study area were $7.729 \pm 0.934 \mathrm{mg} \cdot \mathrm{kg}^{-1}$ and $33.377 \%$ during the dry season (Table 1, Fig. 6). The mean concentration (7.753 \pm 3.070 $\left.\mathrm{mg} \cdot \mathrm{kg}^{-1}\right)$ and \% CV (39.599\%) were obtained in leaves of fluted pumpkin during wet season (Table 2, Fig. 6). The total average concentration of Mn for dry and wet seasons was $7.741 \mathrm{mg} \cdot \mathrm{kg}^{-1}$ (Table 3) - higher than the $4.00 \mathrm{mg} \cdot \mathrm{kg}^{-1}$ in control and the WHO standard of $\left(6.0 \mathrm{mg} \cdot \mathrm{kg}^{-1}\right)$ for $\mathrm{Mn}$ in vegetables. The highest concentration of $12.987 \mathrm{mg} \cdot \mathrm{kg}^{-1}$ was recorded in location 11 (Inua Eyet Ikot). Essiett et al. [17] reported a mean concentration of $208.35 \mathrm{mg} \cdot \mathrm{kg}^{-1}$ of Mn. In a similar work, Nwajei et al. [19] reported concentrations of $4.51 \pm 2.05 \mathrm{mg} \cdot \mathrm{kg}^{-1}$ of $\mathrm{Mn}$ in tomato leaves and $4.51 \pm 0.52 \mathrm{mg} \cdot \mathrm{kg}^{-1}$ of $\mathrm{Mn}$ in tomato fruit samples grown in the study area. Harmanescu et al. [23], stated that Cucumber plants accumulate less Fe but more Mn, $\mathrm{Zn}$, and $\mathrm{Cu}$ in contaminated sites. Manganese acts on the basal metabolism of plants (photosynthesis and transfer of phosphate), stabilizing the structure of the chloroplast and nucleic acid synthesis. There is an apprehension that industrial emissions of $\mathrm{Mn}$ and the use of Mn-containing compounds such as fuel additives may increase the population risk of Parkinson's disease-like disorders. A Canadian study suggested that exposure to ambient Mn concentration advances the age of diagnosis of Parkinson's disease, coherent with the hypothesis that exposure to $\mathrm{Mn}$ adds to the usual loss of neurons attributable to the aging process [28].

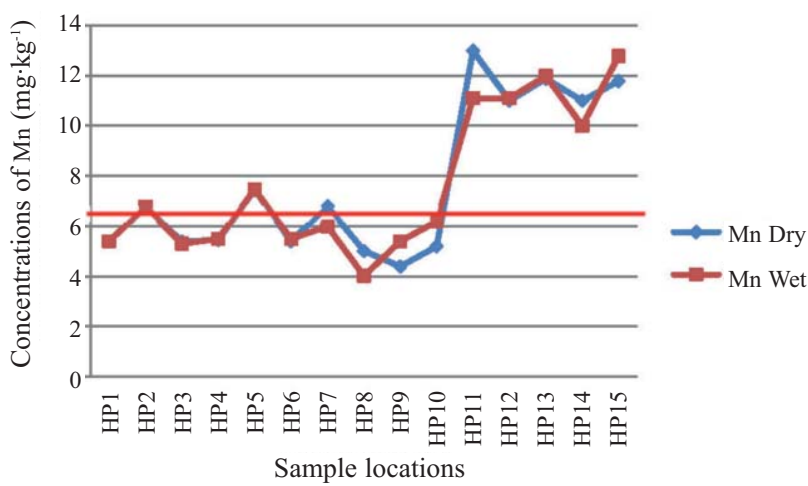

Fig. 6. Dry and wet season variations in concentrations of $\mathrm{Mn}$ in leaves of Telfairia occidentalis with WHO threshold. 


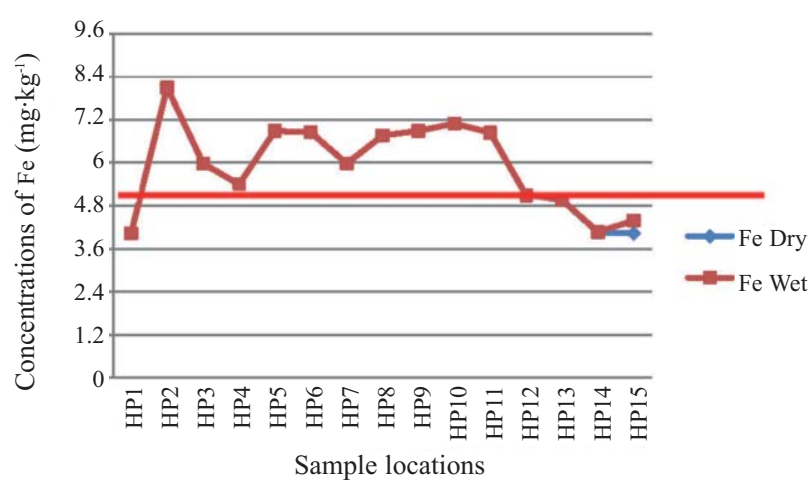

Fig. 7. Dry and wet season variations in concentrations of $\mathrm{Fe}$ in leaves of Telfairia occidentalis with WHO threshold.

\section{$\operatorname{Iron}(\mathrm{Fe})$}

Mean concentration $\left(5.929 \pm 1.279 \mathrm{mg} \cdot \mathrm{kg}^{-1}\right)$ and \% CV $(21.575 \%)$ of $\mathrm{Fe}$ in leaves of Telfairia occidentalis samples were recorded in the dry season (Table 1). Also, during the wet season the mean concentration of $5.955 \pm 1.24 \mathrm{mg} \cdot \mathrm{kg}^{-1}$ and \% CV (20.872\%) were obtained (Table 3). Total average of $\mathrm{Fe}$ for wet and dry seasons was $5.943 \mathrm{mg} \cdot \mathrm{kg}^{-1}$ (Table 3) .The highest concentration of $8.095 \mathrm{mg} \cdot \mathrm{kg}^{-1}$ was recorded in location 2 (Ntafre) and the lowest concentration of $4.029 \mathrm{mg} \cdot \mathrm{kg}^{-1}$ in location 15 (Mkpanak) during the dry season (Fig. 7). The total average concentration (5.94 $\left.\mathrm{mg} \cdot \mathrm{kg}^{-1}\right)$ of Fe in leaves of fluted pumpkin was high when compared with the control sample $\left(3.01 \mathrm{mg} \cdot \mathrm{kg}^{-1}\right)$ and WHO standard $\left(4.0 \mathrm{mg} \cdot \mathrm{kg}^{-1}\right)$ for $\mathrm{Fe}$ in vegetables. Idodo-Umeh et al. [10] reported a mean concentration of $4.865 \mathrm{mg} \cdot \mathrm{kg}^{-1} \mathrm{Fe}$ in cassava tubers in the study area. Also, Nwajei et al. [19] reported concentrations of $7.13 \pm 0.45 \mathrm{mg} \cdot \mathrm{kg}^{-1}$ and $6.00 \pm 0.14 \mathrm{mg} \cdot \mathrm{kg}^{-1}$ of $\mathrm{Mn}$ in tomato leaves and $4.51 \pm 0.52$ $\mathrm{mg} \cdot \mathrm{kg}^{-1}$ of $\mathrm{Fe}$ in tomato leaves and fruit samples grown in the study area. Pumpkin leaves had the highest mean concentration of iron, followed by waterleaf. Humans utilize iron in the hemoglobin of red blood cells in order to transport oxygen from lungs to the tissues. Iron is also an essential component of myoglobin to store and diffuse oxygen in muscle cells. The human body needs iron for oxygen transport. Iron serves vital functions, but as we age excess stores of this metal may build and become toxic. Initially the symptoms are not worrisome but as the iron level keeps on increasing, symptoms like constipation, poor sex drive, fatigue, headache, dizziness, and abdominal cramps become more serious.

\section{Zinc (Zn)}

The mean level of zinc in Telfairia occidentalis leaf samples was $0.031 \pm 0.027 \mathrm{mg} \cdot \mathrm{kg}^{-1}$, and $\% \mathrm{CV}$ of $(86.550 \%)$ was recorded in dry season (Table 1, Fig. 8). Similarly, mean concentration of $\left(0.107 \pm 0.217 \mathrm{mg} \cdot \mathrm{kg}^{-1}\right)$ and $\%$ CV of (202.760\%) of Zn were recorded during wet season (Table 2, Fig. 8). The total average of $\mathrm{Zn}$ for dry and wet seasons was $0.318 \mathrm{mg} \cdot \mathrm{kg}^{-1}$ (Table 3). The highest concentration of $0.071 \mathrm{mg} \cdot \mathrm{kg}^{-1}$ was recorded in location 14
(Okom Ita) and the lowest $-0.007 \mathrm{mg} \cdot \mathrm{kg}^{-1}$ - was recorded in location 7 (Iwo Okpon). The total average concentration of $0.318 \mathrm{mg} \cdot \mathrm{kg}^{-1}$ of $\mathrm{Zn}$ in leaves Telfairia occidentalis was quite low when compared to the concentration of $\mathrm{Zn}$ in control plant samples $\left(0.01 \mathrm{mg} \cdot \mathrm{kg}^{-1}\right)$ and WHO standard $\left(6.0 \mathrm{mg} \cdot \mathrm{kg}^{-1}\right)$. In a similar study, Nwajei et al. [19] recorded a mean concentration of $4.89 \pm 0.5 \mathrm{mg} \cdot \mathrm{kg}^{-1}$ of $\mathrm{Zn}$ in tomato leaves; percentage covariant $(21.47 \%)$ and mean concentration of $\left(3.33 \pm 0.52 \mathrm{mg} \cdot \mathrm{kg}^{-1}\right)$ of $\mathrm{Zn}$ in tomato fruit in the study area. Also, Idoho-Umeh et al. [4] reported a mean concentration of $3.39 \mathrm{mg} \cdot \mathrm{kg}^{-1}$ of $\mathrm{Zn}$ in epicarp of plantain fruit. In "Health risk assessment of heavy metals in seasonal vegetables from northwest Himalaya," Tewari and Pande [21] reported a range of 2.667 to $26.00 \mathrm{mg} \cdot \mathrm{kg}^{-1}$ of $\mathrm{Zn}$ in ladyfinger.

\section{Cobalt (Co)}

The mean concentration of Co in leaves of Telfairia occidentalis samples was $0.101 \pm 0.164 \mathrm{mg} \cdot \mathrm{kg}^{-1}$ and $\% \mathrm{CV}$ $(99.06 \%)$ in the dry season (Table 1, Fig. 9). Also, mean concentrations of $\left(0.0534 \pm 0.023 \mathrm{mg} \cdot \mathrm{kg}^{-1}\right)$ and $\% \mathrm{CV}$ of $43.44 \%$ of $\mathrm{Co}$ in leaves of Telfairia occidentalis samples were recorded during wet (Table 2, Fig 9). The concentration of Co was higher in the dry season compared to wet. Total average concentration of $0.077 \mathrm{mg} \cdot \mathrm{kg}^{-1}$ of Co was obtained for dry and wet seasons (Table 3). Nwajei et al. [19] recorded mean concentration of $0.15 \pm 0.08 \mathrm{mg} \cdot \mathrm{kg}^{-1} \mathrm{Co}$ in tomato leaves samples and mean concentration of $0.05 \pm 0.01 \mathrm{mg} \cdot \mathrm{kg}^{-1}$ in tomato fruits. Essiet et al. [17] recorded a concentration of $1.23 \mathrm{mg} \cdot \mathrm{kg}^{-1}$ of $\mathrm{Co}$ in leaves fluted pumpkin. The accumulation of heavy metal is of great concern in agricultural products owning to the potential threat to human and animal health; heavy metals jeopardize soilplant systems [32].

Cobalt is beneficial for humans because it is a part of vitamin B12, which is essential for human health. Cobalt is used to treat anaemia in pregnant women because it stimulates the production of red blood cells. The total daily intake of cobalt is variable and may be as much as $1 \mathrm{mg}$, but almost all will pass through the body un-adsorbed, except for that in B12. However, too high concentrations of cobalt may damage human health. Health effects that are a result of the uptake of high concentrations of cobalt are: vomiting,

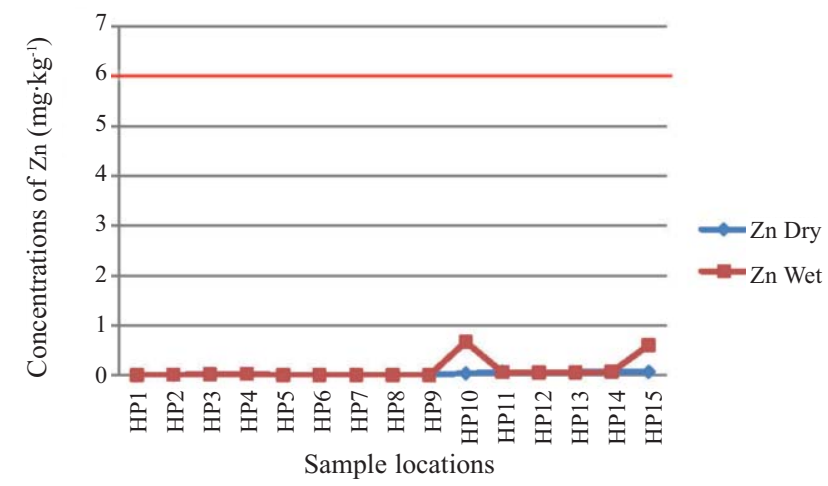

Fig. 8. Dry and wet season variations in concentrations of $\mathrm{Zn}$ in leaves of Telfairia occidentalis with WHO threshold. 


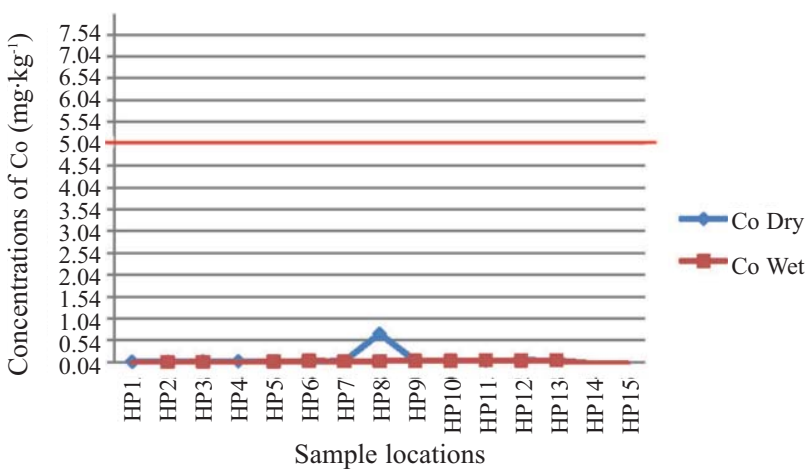

Fig. 9. Dry and wet season variations in concentrations of Co in leaves of Telfairia occidentalis with WHO threshold.

vision problems, heart problems, and thyroid damage [32]. The consumption of some vegetables collected from contaminated sites showed a high capacity for metal accumulation, which further transfers these metals to adults and children. Children ingest significant amounts of metals as compared to adults, but are nearly free of risk. Regularly monitoring these metals in soils, vegetables, and in other food items is necessary to prevent the accumulation of these metals in the food chain [23].

\section{Conclusion}

Concentrations of traces heavy metals in plants are essential for good health and growth of animals and human beings, but the levels should be within permissible limits. As indicated in the results of this investigation, the mean concentrations of $\mathrm{Ni}, \mathrm{V}, \mathrm{Zn}, \mathrm{Co}$, and $\mathrm{Pb}$ were within the normal ranges of concentrations in vegetables as specified by WHO/FAO, the Nigerian Federal Ministry of the Environment, and the German Federal Health Agency Guidelines. The exceptions were mean concentrations of $\mathrm{Cd}, \mathrm{Mn}$, and $\mathrm{Fe}$. As a result, the pollution status of fluted pumpkin in the study area with respect to $\mathrm{Cd}, \mathrm{Mn}$, and $\mathrm{Fe}$ is significant. Consequently, humans and farm animals in the area are under the threat of contamination by these metals. This may bring about possible bio-accumulation in the tissues of various biotas and may in turn threaten the populations of humans and animals that depend on it for food with associated health risk being manifested. Seasons influence the rate of assimilation of heavy metals in fluted pumpkin leaves. Based on the data obtained from this study, it will be important to conduct a subsequent study to assess the levels of the toxic elements for other vegetables that are cultivated and sold in different markets in Ibeno Local Government Area. Findings from such a study in conjunction with the findings that are reported in this study will help to persuade policy makers on the need to identify suitable and non-suitable areas for vegetable production in the area. Management measures to reduce the transfer of heavy metals from historically contaminated soils into the local food chain should be developed and sustained.

\section{Acknowledgements}

The authors wish to thank the staff of post graduate laboratory, Department of Pure and Industrial Chemistry, University of Nigeria, Nsukka for their technical assistance and the provision of equipment and glassware necessary for this work

\section{References}

1. OGBOI E. Spread and Bio Availability of Heavy Metals in Tops Oil and Groundnut (Arachis hypogaeal) in Crude Oil Polluted area of Delta State. Journal of Agriculture and Veterinary Sciences. 4, 85, 2012. www.cenresinpub.org

2. RADMILA N. P., ALEKSANDRA B. S., DRAGANA J. J. Assessment of Soil and Plant Contamination by Select Heavy Metals Along a Major European Highway. Pol. J. Environ. Stud. 22, (5), 1465, 2013.

3. OLADUNNI B. O., TIJUMADE A., OTOLORIN A. O. Heavy Contamination of Water, Soil and Plants around an Electronic waste Dumpsite. Pol. J. Environ. Stud. 22, (5), 1431, 2013.

4. AGBOGIDI O.M. Trace metal profile of some fruits in Kokori and Abraka Market, Delta State, Nigeria. International Journal of Scholarly Research Gate. 2, (1), 4, 2014.

5. KIHAMPA C., MWEGOHA W. J.S., SHEMDOE R. S. Heavy metals concentrations in vegetables grown in the vicinity of the closed dumpsite. International Journal of Environmental Sciences 2, 889, 2011.

6. OSU CHARLES I., OGOKO E.C. Bioconcentration and Transfer of Heavy Metal from Soil into Verninia amydalina, Telfera occidendalis, and Amarathus spinosus. Journal of Applied Phytotechnology in Environmental Sanitation, 3, (4), 117, 2014. https://www.trisanita.org/japes.

7. ATHAR R., AHMAD M. Heavy metal toxicity: Effect on plants growth and metal uptake by wheat and on free living Azotobacter. Water, Air and Soil Pollution. Kluwer Academic Publisher. Printed in the Netherlands. 138, 165, 2001.

8. GIDEON-OGERO E. Levels of heavy metals (lead, cadmium, zinc, magnesium, and cupper) in cassava from Niger Delta of Nigeria as an indication of soil Environmental pollution. Nigerian Village Square, http:www.igeriavillagesquare.com. 1-6, 2008.

9. UBOH E., AKPANABIATU M.I., EDET E.E., OKON I.E. Distribution of heavy metals in fluted pumpkin Telfeiria occidentalis) leaves planted at traffic congested high-away. International Journal of Advance Biotechnology and Research. 2, (2), 250, 2011. http://www.biopublication.com.

10. IDODO-UMEH G., OGBEIBU E. Bioaccumulation of heavy metals in cassava tubers and plantain fruits grown in soils impacted with petroleum and non-petroleum activities. Res. J. Environ. Sci. 4, 33, 2010.

11. FIFIELD W., HAINA I. Environmental Analytical Chemistry. Blackie Academic and Professional. Chapman and Hall, Boundary Row, London. 56-60, 1997.

12. Unilag Consult. Environmental impact assessment for the proposed QIT 750TBD (crude) expansion project, Akwa Ibom State, Nigeria, Onshore Facilities, Mobil Producing Nigeria Unlimited, Lagos Nigeria. 12-13, 1997. 
13. RADOJVIC M., BASHKIN V.N. Practical Environmental Analysis. The Royal Society of chemistry. Thomas Graham House, Science Park, Milton Road, Cambridge UK. 405500, 1999.

14. Cordex Alimentarius Commission (Joint FAO/WHO Food Standards Programme Codex Committee on contaminants in Foods) working document for information and use in discussions related to contaminants and toxins in the GSCTFF (Prepared by Japan and the Netherlands) Fifth Session the Hague, Viale delle Terme di Caracalla, 00153Rome, Italywww.cordexalimentarius.net. The Netherlands, 21-25 March 2011.

15. CHOI Y.Y. International/National Standards for Heavy Metals in Food. Codex standards, http://www.codexalimentarius.net/download/standards/17/CXS_193e.pdf. 2011.

16. ALLOWAY J. Processes and the behavior of heavy metals Heavy metal in soil. Blackie and son Limited. Bishop Briggs, Glasgow. 43-56, 1999.

17. ESSIETT U.A., EFFIONG G.S., OGBENUDIA F.O., BRUNO E.J. Heavy metal concentrations in plants growing in crude oil contaminated soil in Akwa Ibom State, Nigeria. African Journal of Pharmacy and Pharmacology 4, (7), 466, 2010. http://www.academicjournals.org/ajpp.

18. EDEM N.E., OBADONI B.O., ERHANI H., OSAKWUMI U.E. Eco-phytochemical studies of plants in a crude oil polluted terrestrial Habitant located at Iwhreken, Ughelli North Local Government Area of Delta State. Nature and Science, 7, (9), 78, 2009.

19. NWAJEI G.E., OKWAGI P., NWAJEI R.I., OBI-IYEKE G.E. Analytical assessment of trace elements in soils, tomato leaves and fruits in the vicinity of Paint industry, Nigeria. Research Journal of Recent Sciences. 1, (4), 22, 2012. http://www.isca.in.

20. SOBUKOLA O.P., ADENIRAN O.M., ODEDAIRO A.A., KAJIHAUSA E.O. Heavy metal levels of some fruits and leafy vegetables from selected markets in Lagos, Nigeria. African Journal of Food Science, 4, (2), 389, 2009.

21. TEWARI G., PANDE C. Health risk assessment of heavy metals in seasonal vegetablesfrom north-west Himalaya. African Journal of Agricultural Research. 8, (23), 3019, 2013.

22. OYEDEJI A. A., ADEBIYI A. O., OMOTOYINBO M. A., OGUNKUNLE C. O. Effect of Crude Oil-Contaminated Soil on Germination and Growth Performance of
Abelmoschus esculentus L.Moench - A Widely Cultivated Vegetable Crop in Nigeria:

http://www.scirp.org/journal/PaperInformation.aspx?Paper $\mathrm{ID}=24148 \#$. American Journal of Plant Sciences, 3, 1451, 2012.

23. HARMANESCU M., ALDA L. M., BORDEAN D. M., GOGOASA I., GERGEN I. Heavy metals health risk assessment for population via consumption of vegetables grown in old mining area; a case study: Banat County, Romania. Chem Cent J. 5, 64, 2011.

24. YADAV S.K. Heavy metals toxicity in plants: An overview on the role of glutathione and phytochelatins in heavy metal stress tolerance of plants. S. Afr. J. Bot. 76, (2), 167, 2010.

25. Agency for Toxic Substances and Disease Registry (ATSDR). Toxicological profile for Vanadium. Atlanta, GA: U.S. Department of Health and Human Services, Public Health Service. 2012.

26. ASAOLU S.S., ASAOLU M.F. Trace metal distribution in Nigerian leafy vegetables. Pakistan Journal of Nutrition. 9, (1), 91, 2010

27. HOWARD I.C., HORSFALL M., SPIFF I.A., TEME S.C. Heavy metal levels in surface water and sediments in an oil field in the Niger Delta, Nigeria. Global Journal of Pure and Applied Science. 12, (10), 79, 2006

28. ADEKUNLE I.M., BAMGBOSE O., ADETUNJI M.T., LANREIYANDA Y.A. Impact of un-remediated solid waste dumpsite converted to Farmland on plant cadmium phytoaccumulation and toxicity. Journal of Applied Sciences in Environmental Sanitation, 5, (3), 239, 2010.

29. ERIC PEREZ M. D. Cobalt poisoning, U.S. National Library of Medicine, 8600 Rockville Pike, Bethesda, MD 20894 U.S. Department Of Health And Human Services National Institutes Of Health 2012.

30. International Cadmium Association, Cadmium exposure and human health, http://www.cadmium.org/news.php?id news $=8,2013$.

31. OPAJOBI A.O. I., ESUME C.O., OSASUYI A., OKEHIE C.C. Determination of the Lead Content of Pumpkin Leaf Telfairia occidentalis in Selected Towns of Delta State Covering the three (3) Senatorial Districts of the State Curr World Environ: 6, (1), 39, 2011 http://www.cwejournal.org/?p=1242

32. LIU T., LIU B., ZHANG W. Nutrients and Heavy Metals in Biochar Produced by Sewage Sludge Pyrolysis: Its Application in Soil Amendment. Pol. J. Environ. Stud. 23, (1), 271, 2014. 